\title{
Relato de uma experiência de ensino de enfermagem em saúde coletiva: a informática no ensino de vigilância epidemiológica
}

\author{
AN EXPERIENCE OF NURSING EDUCATION IN COLLECTIVE HEALTH: THE USE OF THE INFORMATICS \\ TO TEACH EPIDEMIOLOGICAL SURVEILLANCE \\ EXPERIENCIA DE LA ENSEÑANZA DE ENFERMERÍA EN SALUD COLECTIVA: USO DE LA INFORMÁTICA \\ EN LA ENSEÑANZA DE LA VIGILANCIA EPIDEMIOLÓGICA
}

\section{Lúcia Yasuko Izumi Nichiata ${ }^{1}$, Renata Ferreira Takahashi ${ }^{1}$, Lislaine Aparecida Fracolli ${ }^{1}$, Anna Luiza de Fátima Pinho Lins Gryschek ${ }^{1}$}

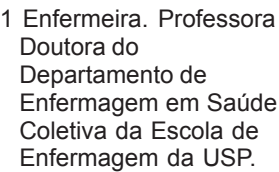

\author{
RESUMO \\ Discute-se a informática \\ na educação, os benefícios, \\ implicações éticas e \\ ideológicas da apropriação \\ desta no ensino de \\ enfermagem e no trabalho \\ do enfermeiro. Relata o \\ desenvolvimento de conteúdo \\ de vigilância epidemiológica \\ na disciplina Enfermagem em \\ Saúde Coletiva com Enfoque \\ nas Doenças Transmissiveis \\ do curso de graduação \\ oferecida em 2000. \\ Utilizando-se do banco de \\ dados de aids, os alunos \\ expressaram sua \\ compreensão sobre os \\ problemas de saúde da \\ população, tomando \\ exemplarmente os casos de \\ aids em São Paulo, \\ associando à indicadores \\ socioeconômicos.
}

\begin{abstract}
This article discusses the application of information technology (IT) in education, its benefits and the ethical and ideological implications in using this tool in nursing education and work. It relates the experience of developing contents on epidemiological surveillance. Some pedagogical experiences were given to the students handling an Aids database, with the purpose of motivating them to express their understanding and to reflect on the health problems of the population, associating the occurrence and the profiles of this epidemic in different areas of the city of São Paulo, with the forms of work and life of population groups in certain places. The understanding of the healthdisease process was enlarged. IT is a potent instrument in epidemiological surveillance for the teacher who act as mediator between the information and the knowledge that one wants to build.
\end{abstract}

\section{KEYWORDS}

PALAVRAS-CHAVE

Vigilância epidemiológica.

Enfermagem.

Informática em saúde.
Epidemiologic surveillance. Nursing. Medical informatics.

\section{RESUMEN}

El presente artículo presenta la discusión sobre las aplicaciones de la informática en la educación y los beneficios e implicancias éticas e ideológicas de la apropiación de esta herramienta, en la enseñanza de enfermería y en el trabajo del enfermero.Relata el desarrollo del contenido sobre vigilancia epidemiológica en la disciplina Enfermería en Salud Colectiva con Enfoque en las Enfermedades Transmisibles del pre grado ofrecida en el 2000. Utilizándose el banco de datos sobre el SIDA, los alumnos fueron incentivados a expresar su comprensión y reflexión sobre los problemas de salud, asociando la ocurrencia y los perfiles de esa epidemia, en São Paulo, con las formas de trabajary vivir. La informática es un instrumento potente en la VE, pues torna de manera ágil el procesamiento y análisis de los datos y posibilita la búsqueda de informaciones en otras fuentes de datos.

\section{PALABRAS-CLAVE}

Vigilancia epidemiológica. Enfermería. Informática Médica. 


\section{INTRODUÇÃO}

A área da saúde tem se beneficiado com a utilização da informática, especialmente na conformação de sistemas de informação demográficos e de saúde de fundamental importância para a prática de vigilância e monitoramento dos agravos e indicadores de morbidade e mortalidade.

Especialmente na prática de vigilância epidemiológica, o avanço tecnológico no campo da informática e da comunicação, no início dos anos 80 , vem se constituindo num elemento fundamental de auxílio no processamento e na análise de dados sobre a realidade epidemiológica das populações. Esta nova ciência tem possibilitado a reorganização dos sistemas de informação sobre saúde em geral e especificamente da vigilância epidemiológica. Como exemplo, tem-se a constituição de bancos de dados nacionais de doenças de notificação e de diversos aplicativos de análises estatísticas que vêm proporcionando maior rapidez, agilidade e descentralização na produção da informação em saúde.

Dessa forma, a informação produzida passa a servir de base, não só para a definição e avaliação das próprias ações de vigilância epidemiológica, mas principalmente, ao redirecionamento das ações para a prevenção e o controle das doenças na população.

Considera-se que a produção das informações em saúde, obtida de forma ágil, atualizada, completa e fidedigna, vem se conformando num importante instrumento de controle social, na medida em que possibilita à população o acompanhamento e a avaliação das atividades dos serviços de saúde. Isso também é válido para a análise das prioridades políticas a partir dos perfis epidemiológicos de determinada localidade e, principalmente, na fiscalização da aplicação dos recursos públicos, conformando-se numa estratégia para a operacionalização do Sistema Único de Saúde ${ }^{(1)}$.

Contudo, não se deve perder de vista que o conhecimento sobre o uso desta tecnologia, reconhecidamente um recurso fundamental para a análise de dados epidemiológicos, não pode ser entendida como uma atividade "fim", no sentido de que sua valorização recaia sobre o seu próprio procedimento técnico. A utilização da informática como uma atividade "meio" significa compreendê-la como um potente instrumental para produzir e analisar dados e gerar a informação. Ainda, por meio da informação, intervir no processo saúdedoença em sua dimensão coletiva, contemplando as questões dos paradigmas assistenciais, assim como as questões éticas e sociais mais complexas envolvidas na utilização desta tecnologia.

Entende-se que a incorporação dos recursos da informática, como um instrumento de trabalho, deve ser apreendido pelo Enfermeiro assim como o são o exame físico, a entrevista e o diagnóstico de enfermagem. Há necessidade de incorporar a informática como uma ferramenta de trabalho do Enfermeiro, uma vez que não se pode negar as revoluções no mundo do trabalho que esta área do conhecimento têm trazido.

Este trabalho tem por finalidade discutir a importância da informática no ensino da Enfermagem, particularmente no desenvolvimento do conteúdo de vigilância epidemio-lógica e relatar a experiência da disciplina Enfermagem em Saúde Coletiva com Enfoque nas Doenças Transmissíveis (ESC-DT) da Escola de Enfermagem da Universidade de São Paulo (EEUSP) na utilização da informática como um instrumento pedagógico.

\section{A INFORMÁTICA E A EDUCAÇÃO}

Passados mais de 50 anos da criação do primeiro computador na Universidade de Harvard, Estados Unidos, é fato que estamos vivendo neste início de século a era da informática. Inicialmente desenvolvida com o intuito de responder às necessidades e finalidades bélicas e militares, a aplicação da informática hoje se estende a praticamente todas as áreas do conhecimento ${ }^{(2)}$. Concebem-se três maneiras em que a informática tem sido utilizada na educação: a primeira é ela própria objeto de estudo, em que o aluno estuda a área de conhecimento da informática. A segunda maneira, quando a informática é utilizada para ensinar os diversos usos dos seus recursos, por exemplo, através de cursos de noções básicas e aplicações dos computadores e Internet. Em terceiro lugar, utilizada para promover a aprendizagem em outras áreas do conhecimento como Matemática, Português, Ciências, etc.. ${ }^{(2)}$.

Seja qual for a situação, diversos autores chamam a atenção para as modificações que
Relato de uma experiência de ensino de enfermagem em saúde coletiva: a informática no ensino de vigilância epidemiológica 
Lúcia Yasuko I. Nichiata Renata Ferreira Takahashi Lislaine Aparecida Fracolli Anna Luiza de F.P.L.Gyschek

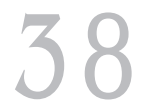

Rev Esc Enferm USP 2003; 37(3): 36-43. os recursos da informática trouxeram a uma nova estrutura de educação ${ }^{(3-4)}$. Considerase que de todas as tecnologias de comunicação desenvolvidas até o momento, desde a imprensa escrita, o rádio e a televisão, o computador seja aquela que elimina a necessidade de se ir a um lugar específico. De fato, talvez não seja mais necessário haver salas de aula como elas existem atualmente. Alguns conteúdos já são desenvolvidos de forma descentralizada, através dos recursos de multimídia, como os cursos on-line à distância e as vídeo-conferências, com a possibilidade a qualquer tempo de revisão dos conhecimentos adquiridos.

A informática também trouxe modificações nas tradicionais relações de poder entre professor-aluno, pelo fato de que os mestres não são mais os únicos e sábios fornecedores do conhecimento, portanto, detentores de um saber universal. São agora, agentes do conhecimento, catalisadores no processo de aprendizagem $^{(4)}$. Outro aspecto considerado favorável ao uso da tecnologia de informática é que o currículo pode ser flexibilizado, integrando disciplinas e variadas áreas do conhecimento. Esta seria a principal implicação do uso da informática na educação, elaborando um currículo que acentua a integração das disciplinas ao invés de separá-las. Argumenta-se que esta não seria uma razão para aprender, por exemplo, Literatura Inglesa e História Inglesa em dois cursos e horários distintos, pois com a informática estes conhecimentos podem ser reunidos a qualquer tempo e lugar, pelo próprio aluno ${ }^{(2)}$.

Dentre os argumentos que falam a favor da utilização da informática no ensino, destacam-se ainda os estudos de avaliação do ensino assistido por computador. De um modo geral, estes estudos apontam a redução substancial do tempo gasto na aprendizagem pelos alunos, quando comparado ao uso de estratégias tradicionais de ensino; o aumento das notas de avaliação e o desenvolvimento de atitudes positivas em relação às atividades propostas, demonstram resultados positivos quanto à avaliação ${ }^{(2-4)}$. Segundo Barros e D’Ambrósio (1998) o uso do computador é por si só uma motivação para o aprendizado, além dos desafios para superar os problemas estipulados, através de exercícios de simulação. Outras aplicações da informática na educação podem ser apontadas, entre elas sua contribuição como um instrumento de acompanhamento das atividades administrativas da escola (manutenção de registros sobre os alunos, corpo docente, organização de bancos de dados e catalogação de livros), bem como do desempenho dos alunos (cálculo e avaliação das taxas de reprovação e aprovação).

O avanço desta tecnologia nas diferentes áreas do conhecimento é evidente e a passagem pelo sistema educacional deve ser beneficiada pelas vantagens de sua apropriação. Entretanto, é preciso analisar com cautela a visão que comumente vem sendo colocada, de que a tecnologia é algo "redentor" da pedagogia. ${ }^{(4)} \mathrm{A}$ tecnologia não é um produto autônomo, como se tivesse vida própria, independente das relações sociais estabelecidas para o seu desenvolvimento. É preciso relativizar a idéia de que ela veio mudar radicalmente nossas vidas nas escolas. A informática vem sendo incorporada pela sociedade em geral como um meio para aumentar cada vez mais o bem-estar da população, entretanto, no atual desenvolvimento da sociedade brasileira, pode-se concordar que isso é parcialmente verdadeiro. Se a tecnologia é criada para mudar nossas vidas, no sentido da melhoria de sua qualidade, que relações permanecem as mesmas? O computador contribui para melhorar a vida de quem? Quem tem acesso a esse bem? Quem decide quando e onde a informática será utilizada? O foco crescente na especialização tecnológica, particularmente nas habilidades de uso do computador na área da saúde, estará a serviço de tornar mais igualitárias as relações sociais ou exacerbará ainda mais as desigualdades e a exclusão social em nosso meio? O que significa para o processo escolar e para a crença, que parece largamente difundida, de que o futuro mundo do trabalho exigirá uma competência técnica crescente por parte de todos os profissionais?

Estas questões nos remetem às discussões éticas e ideológicas relacionadas a que necessidades sociais as escolas de enfermagem devem responder e aos interesses de quem elas devem servir, indagações estas nada fáceis de serem respondidas. Entretanto, não podem ser ignoradas, se o interesse é ter um enfermeiro consciente do seu papel social, em defesa da saúde coletiva e crítico em relação à realidade de saúde brasileira. No ensino de enfermagem, apropriar-se dos instrumentais da informática deve significar o 
desenvolvimento de atitudes e disposição socio-motivacionais relacionados ao trabalho em saúde, no que diz respeito a responsabilidade em relação à transformação dessa realidade.

\section{A APLICAÇÃO DA INFORMÁTICA NO ENSINO DE ENFERMAGEM}

As primeiras aplicações da informática no ensino de enfermagem no Brasil, foram desenvolvidas nas Universidades e Escolas Públicas de Enfermagem, na década de 80. Visavam desenvolver recursos nos processos de ensino-aprendizagem, instrumentalizando os alunos a utilizarem os computadores como instrumentos na elaboração de pesquisas, editoração de textos, construção de bancos de dados e pesquisas em bibliotecas ${ }^{(5-7)}$.

Mais recentemente a informática como um recurso auxiliar no ensino de enfermagem, vem se desenvolvendo com a construção dos sistemas de computação aplicados ao ensino ${ }^{(7-8)}$. Os programas de simulações computa-dorizados podem aproximar o ensino teórico ao prático, estimulando o aluno a solucionar os problemas colocados.

De um modo geral, os entusiastas da informatização do ensino de enfermagem defendem a importância de maiores investimentos nesta área, uma vez que o setor de educação não pode ficar à margem do atual processo de desenvolvimento tecnológico.

Os trabalhos que tratam da utilização da informática na enfermagem, sejam no ensino, na pesquisa ou na assistência, abordam invariavelmente mais o aspecto do "como" - visando tornar o usuário (aluno, enfermeiro) tecnicamente competente para o seu uso - em detrimento do "porquê" - no sentido de discutir as questões em torno dos impactos sociais mais amplos que a nova tecnologia traz. A utilização da informática no ensino de Enfermagem em Saúde Coletiva é necessária aos alunos, dadas as peculiaridades do trabalho neste campo científico. Historicamente, trabalhar com dimensões coletivas da saúde pressupõe o uso de conhecimentos, dentre outros, advindos dos campos da Epidemiologia, da Estatística e da Matemática, os quais se apropriaram grandemente das tecnologias da informática e da informação. A informatização na área da saúde é um componente do sistema de informação que subsidia em última instância, o acompanhamento dos perfis epidemiológicos das coletividades, visando a melhoria das condições de vida da população ${ }^{(1,8-10)}$.

Assim, a utilização da informática no processo de ensino-aprendizagem de Enfermagem em Saúde Coletiva é mais do que um facilitador pedagógico, constitui-se em um instrumento de trabalho para o enfermeiro que irá atuar em diferentes territórios e coletividades. Numa discussão mais ampla sobre as necessidades de transformação do trabalho do enfermeiro, apontou-se como desafios para a enfermagem nos anos 90 a necessidade de recriar modelos de processos de trabalho em enfermagem que contemplem objetos e finalidades sociais a partir da finalidade do processo de trabalho em saúde ${ }^{(9)}$.

Cabe à Enfermagem criar ou apropriar-se de instrumentos que permitam intervir na dimensão coletiva do processo saúde-doença e recriar relações mais democráticas com os usuários dos serviços de saúde, com os trabalhadores da equipe de enfermagem e com os demais trabalhadores dessa área, fundamentais para o alcance dos princípios e diretrizes do Sistema Único de Saúde ${ }^{(9)}$. Estas proposições não devem ser entendidas nem como responsabilidade única da enfermagem e muito menos como algo que, no presente momento a enfermagem é a mais potente para deflagrar. Apenas que é necessário fazer enquanto parte significativa do conjunto dos trabalhadores e parte inequívoca do sistema de produção em saúde.

\section{O ENSINO DOS CONTEÚDOS DE VIGILÂNCIA EPIDEMIOLÓGICA E A INFORMÁTICA}

A vigilância epidemiológica, descrita como

conjunto de ações que proporcionam o conhecimento, a detecção e prevenção de qualquer mudança nos fatores determinantes e condicionantes de saúde individual e coletiva, com a finalidade de recomendar e adotar as medidas de prevenção e controle das doenças e agravos,

implica a organização de um sistema de informação para a obtenção de dados que tenham utilidade na previsão de eventos ligados à saúde, de maneira que ações efetivas de prevenção e controle possam ser implementadas ${ }^{(1)}$.
Relato de uma experiência de ensino de enfermagem em saúde coletiva: a informática no ensino de vigilância epidemiológica 
Lúcia Yasuko I. Nichiata Renata Ferreira Takahashi Lislaine Aparecida Fracolli Anna Luiza de F.P.L.Gyschek

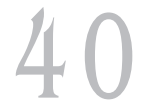

Rev Esc Enferm USP 2003; 37(3): 36-43.
A vigilância epidemiológica é composta basicamente das seguintes atividades: a) coleta e processamento dos dados, b) análise e interpretação de dados, c) disseminação das informações produzidas e recomendação das medidas de controle apropriadas, d) promoção das ações de controle, e) avaliação das medidas adotadas e retroalimentação das informações produzidas.

Desde o início da década de sessenta, culminando na sua criação em 1975, a vigilância epidemiológica vem sofrendo modificações na sua definição e prática. Com a conformação do Sistema Único de Saúde (SUS) no Brasil, a descentralização da produção das informações em saúde - uma das diretrizes de atuação político-administrativa - vem demandando que os municípios assumam a responsabilidade pelo desenvolvimento da vigilância epidemiológica de sua região geopolítica $^{(1,11)}$.

Os sistemas de informação produzem um grande volume de dados e cada vez mais exigese que o sistema seja capaz de receber, arquivar e distribuir dados de maneira rápida e eficiente. A única possibilidade de viabilização de um sistema de informação com essas características é a utilização das tecnologias de informática, que, ao serem implementadas, tornaram obsoletas as formas manuais de processamento de dados utilizadas anteriormente.

Num claro processo de adesão aos recursos da informática, o Ministério da Saúde vem desenvolvendo, desde a década de 90, esforços no sentido de construir Sistemas Nacionais de Informação. Pode-se citar como exemplo os Sistemas de Informação que fazem parte da Rede Integrada de Informações para a Saúde (RIPSA): Sistema de Informação do Programa de Agentes Comunitários de Saúde (SIPACS), Sistema de informação de Atenção Básica (SIAB), Sistema de Sistema de Informações sobre Mortalidade (SIM), Sistema de Informações sobre Nascidos Vivos (SINASC), Sistema de Informações Ambulatoriais do Sistema Único de Saúde (SIA/SUS), Sistema de Informações Hospitalares (SIH/SUS), Sistema de Informações de Vigilância Alimentar e Nutricional (SISVAN), o Sistema Nacional de Imunizações (SI-PNI) e o Programa SINAN - Sistema de Informações de Agravos de Notificação Compulsória, criado com o propósito de funcionar como um banco que armazena dados relativos às doenças transmissíveis de notificação com- pulsória. Este sistema permite que cada uma das esferas de gestão do sistema de saúde brasileiro realize as análises epidemiológicas, compatíveis com suas atribuições ${ }^{(11-12)}$. Tendo em vista este contexto, a informática, enquanto um instrumento que viabiliza a vigilância epidemiológica nas diversas esferas de gestão, deve ser tomada como um conteúdo de ensino nas disciplinas que se propõem a construir conhecimentos na área de vigilância epidemiológica.

A disciplina Enfermagem em Saúde Coletiva com Enfoque nas Doenças Transmissíveis (ESC-DT), desenvolvida em 2000, junto ao Departamento de Enfermagem em Saúde Coletiva da Escola de Enfermagem da Universidade de São Paulo (EEUSP), teve como propósito permitir que os estudantes expressassem sua compreensão e refletissem sobre os problemas de saúde da população, especialmente aqueles relacionados à ocorrência das doenças transmissíveis, considerando que não há aleatoriedade na disseminação e distribuição das doenças transmissíveis, eventos claramente ligados às condições socioeconômicas e culturais.

Utilizando-se principalmente dos saberes advindos da epidemiologia, das ciências sociais, da biologia e da informática, ESC-DT buscou propiciar experiências pedagógicas capazes de levar os alunos a realizarem práticas simuladas de vigilância epidemiológica, através da análise da ocorrência e distribuição de um dado agravo. A aids no município de São Paulo foi escolhida pois o Programa de DST/Aids da Secretaria de Estado de São Paulo organiza, desde o início da década de 80 um banco de dados informatizado de todos os casos ocorridos na cidade, segundo os distritos administrativos de residência.

Para serem analisados pelos alunos foram selecionados distritos administrativos que guardavam diferenças importantes do ponto de vista de condições de vida das pessoas moradoras de cada região. A seleção dos distritos teve por base o trabalho de Aldaísa Spozatti ${ }^{(13)}$, que analisou as desigualdades sociais, segundo variáveis socioeconômicas. Seu estudo utilizou 141 bases de dados de diferentes fontes de informação, dados estes organizados em quatro indicadores de exclusão: indicador de exclusão de autonomia, indicador de exclusão de desenvolvimento humano, indicador de exclusão de qualidade de vida e indicador de 
exclusão de eqüidade. Os valores calculados em cada um dos quatro indicadores foram convertidos em números de uma escala que variou de -1 (maior nível de exclusão) a +1 (maior nível de inclusão), sendo o valor zero, o padrão básico, de tal forma que os valores positivos mantêm uma relação indissociável com os negativos.

No mapa abaixo, estão representados os 96 distritos administrativos que compõem o município de São Paulo. Distribuindo os distritos, segundo os índices de exclusão em cinco regiões (A, B, C, D e E), pode-se observar que quase a totalidade deles apresenta uma coloração que gradativamente é mais escura no sentido do centro para a periferia, indi- cando graus de exclusão crescente. No centro da cidade encontram-se alguns distritos com melhores indicadores sociais de inclusão, classificados como A (12 de um total de 96 distritos). São os distritos de Moema, Alto de Pinheiros, Jardim Paulista, Santo Amaro, Campo Belo, Vila Mariana, Perdizes, Itaim Bibi, Butantã, Santana, Saúde e Pinheiros. No centro expandido, região B, encontram-se alguns distritos do Centro Velho da cidade, como a Bela Vista, o Cambucí, a Barra Funda, a Móoca e outros. Dentre os 73 distritos com condições de vida mais precárias, regiões $\mathrm{C}, \mathrm{D}$ e E, 22 encontram-se localizados nas periferias mais distantes (Vila Jacui, Cidade Dutra, Brasilândia, Itaquera, Cidade Ademar, Marsilac e outros).

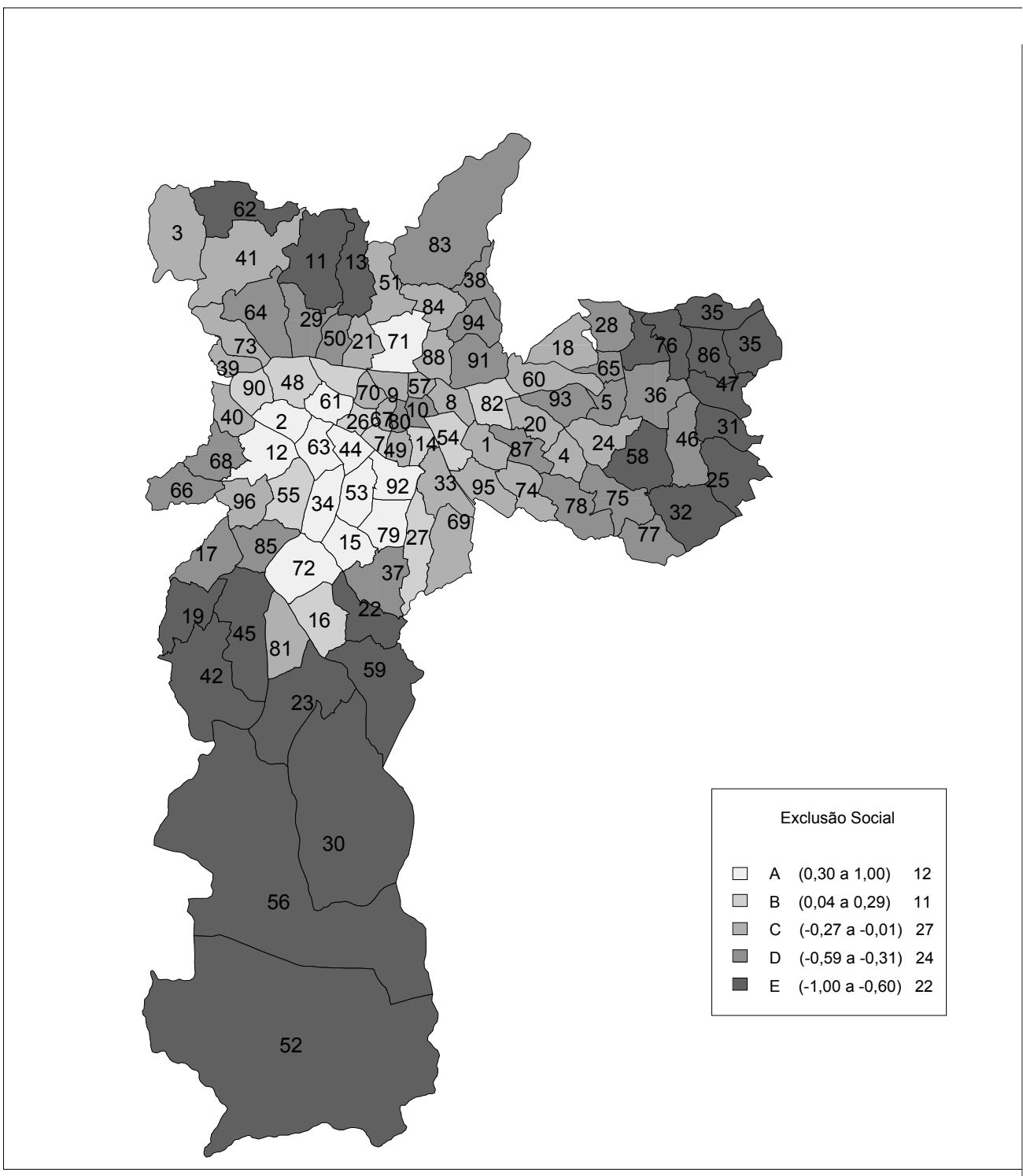

Figura 1: Distribuição dos distritos administrativos, segundo o grau de exclusão/ inclusão $^{(13)}$. (Município de São Paulo, 1996)

Relato de uma experiência de ensino de enfermagem em saúde coletiva: a informática no ensino de vigilância epidemiológica 
Lúcia Yasuko I. Nichiata Renata Ferreira Takahashi Lislaine Aparecida Fracolli Anna Luiza de F.P.L.Gryschek

(a) Projeto "Método interativo no ensino de enfermagem em saúde coletiva com enfoque no controle da Aids", desenvolvido com recursos provenientes do programa Sistema Integrado de Apoio ao Ensino - SIAE da PróReitoria de Graduação Pós-Graduação da Universidade de São Paulo - 1999. Disponível no web-site < htp:// www. ee.usp.br/ graduacao/ens0218/ ens0218.html >

(b) Disponível no web site $<$ http://www.ee.usp.br/ graduacao/ens0435.html.>

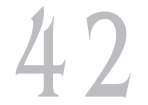

Rev Esc Enferm USP 2003; 37(3): 36-43
Para desenvolverem o exercício proposto pela disciplina, foram selecionados os distritos de Moema (identificado no mapa pelo número 53) e Bela Vista (número 7) de maior inclusão e, Itaquera (número 36) e Brasilândia (número 11), estes dois classificados como distritos de maior exclusão social.

A atividade pedagógica desenvolvida consistiu na descrição e análise da evolução da epidemia nos distritos considerados, mediante a utilização do programa Epi-Info, um pacote estatístico de suporte para a análise dos dados, de domínio público comumente utilizado pelos técnicos da Secretaria de Estado da Saúde na análise de dados. As etapas de análise da distribuição dos casos de aids notificados nos distritos selecionados consistiram em:

Descrição da epidemia: descrição das características dos casos. Realização das freqüências dos dados contidos nas fichas de notificação (idade, faixa etária, sexo, ano de notificação, ano de diagnóstico, ocupação, escolaridade, informação sobre ocorrência do óbito, categoria de exposição ao vírus, presença de doenças oportunistas). Seleção de pelo menos duas variáveis para cruzamento dos dados (ano de diagnóstico x sexo, ano de diagnóstico $\mathrm{x}$ faixa etária, ano de diagnóstico $x$ ano de óbito, sexo x ocupação, etc). Identificação dos resultados que se destacaram.

Análise dos casos de aids: associação da ocorrência da epidemia às condições de vida dos moradores dessas regiões. Nesta etapa foi feito uma comparação dos resultados obtidos nos diferentes distritos, apontando as diferenças em termos do perfil da epidemia em cada região e as características socioeconômicas analisadas no estudo de exclusão/inclusão ${ }^{(13)}$ da cidade para essas localidades.

O exercício proposto possibilitou o reconhecimento da distribuição do agravo em São Paulo e a sua diferenciação por região, conforme se avalia o perfil socioeconômico dos doentes e os indicadores de condições de vida nos diferentes distritos da cidade. Grangeiro ${ }^{(14)}$ analisando o perfil socio-econômico dos casos de aids no início da epidemia já havia demonstrado que a epidemia no município de São Paulo possui um perfil vinculado à heterogeneidade social, peculiar à própria estrutura da cidade. Como síntese do exercício desenvolvido discutiu-se com os alu- nos sobre a necessidade de políticas preventivas e assistenciais que considerem as diferenças que cada segmento social possui em relação às desigualdades encontradas.

Para o desenvolvimento do conteúdo e habilidades, tivemos o apoio do Setor de Informática da Escola que forneceu capacitação específica aos alunos para o manejo deste programa. Os alunos tiveram a oportunidade de utilizar tecnologias de informática, como os componentes de hardware (microcomputadores, rede) e os componentes de software (sistema operacional Windows NT-server, bancos de dados Dbase e Epi-Info, processadores de texto e planilhas de cálculo). Foi indicado como material informativo de apoio os textos sobre VE da aids ${ }^{(a)}$, produzidos em parceria com técnicos do Serviço de Vigilância Epidemiológica do Centro de Referência de DST/Aids e do Programa de DST/ Aids da Secretaria de Estado da Saúde de São Paulo. Além deste, também foi indicado o site que trata da $\mathrm{VE}^{(\mathrm{b})}$, criado também pelo Departamento de Enfermagem em Saúde Coletiva em conjunto com a Secretaria de Estado da Saúde de São Paulo. Incentivou-se ainda a busca de informações em outros sites de interesse, tais como o do Programa Nacional de DST/Aids.

\section{CONSIDERAÇÕES FINAIS}

A informática é uma tecnologia que vem sendo utilizada crescentemente em diferentes campos científicos e profissionais e nesse sentido a enfermagem pode se beneficiar da incorporação desse instrumento ao seu trabalho. Atualmente, a acessibilidade a hardwares e softwares tem sido ampliada, através de equipamentos de fácil operação e da disponibilização de programas na rede mundial de computadores.

Os atuais enfermeiros devem estar atentos às vantagens que o computador oferece para o seu trabalho, tais como o acesso às informações com grande rapidez, desburocratização do serviço e auxílio às tomadas de decisões. Considera-se ainda que as características instrucionais dos computadores propiciam melhores condições de ensino e aprendizagem.

Neste relato os docentes utilizaram os recursos da informática no ensino de vigilância epidemiológica, pois esta prática de saúde pública, vem cada vez mais fazendo uso 
dessa tecnologia em seu cotidiano de trabalho. Buscou-se, através desta experiência de ensino, demonstrar que a informática é um instrumento potente na vigilância epidemiológica, por tornar mais ágil o processamento e análise dos dados e por possibilitar a busca por informações em outras fontes.

Evidenciou-se que o uso da informática na escola facilita a aquisição de informações, contudo o papel social da educação não é meramente ser uma agência transmissora de informação e sim transformar-se em um lugar de análise crítica e produção de informações, onde o conhecimento possibilita a atribuição de significado às informações ${ }^{(6)}$.
$\mathrm{Na}$ presente experiência de ensino, os docentes assumiram um papel de mediadores entre a informação e o conhecimento que se quer construir, papel este facilitado pelo uso da informática. O maior investimento do corpo docente foi de desenvolver junto aos alunos não só a busca e a seleção das informações, como principalmente o saber pensar de modo reflexivo.

\section{AGRADECIMENTOS}

À Equipe de Vigilância Epidemiológica do Programa de DST/Aids da Secretaria de Estado da Saúde de São Paulo, Médica Naila Janilde Seabra Santos e Enfermeira Sirlene Caminada, pelo apoio durante o processo de desenvolvimento da disciplina.

\section{REFERÊNCIAS}

(1) Tasca R, Greco C, Villarosa FN. Sistemas de informação em saúde para distritos sanitários. In: Mendes EV, organizador. Distrito sanitário: o processo social de mudança das práticas sanitárias do Sistema Único de Saúde. São Paulo: Hucitec 1994.

(2) Hocking J. The computer in higher education: not just another mechanical device. Educ Technol 1985; 25(8):19-26.

(3) Barros JPD, D'Ámbrosio U. Computadores, escola e sociedade. São Paulo: Scipione; 1998. (Coleção Informática \& Educação).

(4) Libâneo JC. Adeus professor, adeus professora?: novas exigências educacionais e profissão docente. 4.ed São Paulo: Cortez; 2000. (Coleção Questões da Nossa Época; v.67).

(5) Marin HF. Informática em enfermagem. São Paulo: EPU; 1995.

(6) Évora YDM. Processo de informatização em enfermagem: orientações básicas. São Paulo: EPU; 1995.

(7) Évora YDM. Enfermagem e informática: tendências atuais e perspectivas futuras [tese] Ribeirão Preto (SP): Escola de Enfermagem de Ribeirão Preto/USP; 1993.
(8) Almeida MF. Descentralização de sistemas de informação e o uso das informações a nível municipal. Inf Epidemiol SUS 1998; 7(2):27-33.

(9) Oliveira MAC, Bertolozzi MR, Egry EY, Fonseca RMGS. Globalização e saúde: desafios para a enfermagem em saúde coletiva no limiar do terceiro milênio. Saúde Soc 1998; 7(2):3-18.

(10) Moraes IHS, Santos SRFR. Informação em saúde: os desafios continuam. Ci \& Saúde Coletiva 1998; 3(1):37-51.

(11) Padilha HP, Costa CHL. O plano diretor de informações do Ministério da Saúde: uma estratégia para sua elaboração. Inf Epidemiol SUS 1996; 2:61-6.

(12) Carvalho Adeo, Eduardo MBP. Sistemas de Informação em Saúde para Municípios. São Paulo: FSP/USP/IDS 1998. (Série Saúde \& Cidadania).

(13) Sposati A, organizador. Mapa da exclusão/ inclusão social da cidade de São Paulo. São Paulo: Educ; 1996.

(14) Grangeiro A. O perfil socioeconômico dos casos de aids da Cidade de São Paulo. In: Parker R, Bastos C, Galvão J, Pedrosa JS, organizador. AAIDS no Brasil. Rio de Janeiro: Relume-Dumará; 1994. cap 4, p.91-125.
Relato de uma experiência de ensino de enfermagem em saúde coletiva: a informática no ensino de vigilância epidemiológica
Recebido: 30/04/2002 Aprovado: 01/07/2003 